\title{
Gas Sensors Based on Metal Oxides Decorated by Reduced Graphene Oxide with Enhanced Gas Sensing Properties
}

\author{
Yuan Gao*, Bo Zhang, Ming Cheng, Lianjing Zhao, Geyu Lu* \\ State Key Laboratory on Integrated Optoelectronics, College of Electronic Science and Engineering, \\ Jilin University, 2699 Qianjin Street, Changchun 130012, China \\ gaoyuan@jlu.edu.cn, lugy@jlu.edu.cn
}

\begin{abstract}
:
Sensors based on graphene-derived materials have drawn a great deal of attention for the excellent properties of graphene. Theoretically, graphene can detect toxic gases on molecule level. However, its application in actual gas sensors is limited due to the low and irreversible response. Therefore, we synthesized a series of hybrids of metal oxide and reduced graphene oxide ( $\mathrm{rGO}$ ), including $\mathrm{SnO}_{2} / \mathrm{rGO}$, $\mathrm{ZnO} / \mathrm{rGO}, \mathrm{In}_{2} \mathrm{O}_{3} / \mathrm{rGO}, \alpha-\mathrm{Fe}_{2} \mathrm{O}_{3} / \mathrm{rGO}$, and $\mathrm{Co}_{3} \mathrm{O}_{4} / \mathrm{rGO}$, in which metal oxides were the main components while rGO was employed as decorations. The metal oxides decorated by rGO exhibited enhanced gas sensing properties, especially towards $\mathrm{NO}_{2}$. With optimization, some gas sensors could even work at room temperature, which led to lower energy consumption. As proven in our experiments, the dosages and dispersions of rGO as well as the species and morphologies of metal oxides in hybrids accounted for their gas sensing properties. Specially, rGO played a crucial role in hybrids for response enhancement as its chemical and electronic sensitization. The extra gas absorption and local heterojunctions provided by rGO could also be responsible for the improved sensing properties. These explorations are of exemplary significance for other researchers to develop rGO-based sensitive materials with excellent gas sensing properties in the future.
\end{abstract}

Key words: metal oxide, graphene, hybrids, gas sensor, heterojunctions.

The synthesis of metal oxide/rGO hybrids

Generally, our metal oxide/rGO hybrids were prepared mainly via hydrothermal method. GO solution with a certain concentration acted as the precursor of rGO. During hydrothermal or solvothermal procedure, GO was reduced to form rGO, and metal oxides formed simultaneously. Most of our metal oxide/rGO hybrids were obtained by one-step thermal treatment. Also, the physically combination was employed for $\mathrm{Co}_{3} \mathrm{O}_{4} / \mathrm{rGO}$. At last, the hybrids were sintered under air or inert atmosphere to futher remove the impurities and also improve their crystallinity and chemical stability. We studied their crystallography, morphology, chemical component, dispersion, and work function by XRD, TEM, SEM, XPS and UPS to find out the relationships with their sensing properties and investigate sensing mechanism in depth.

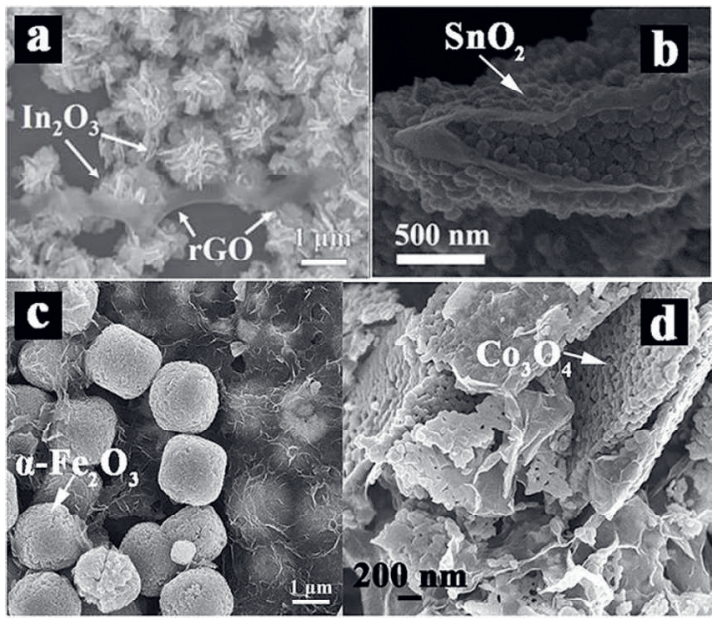

Fig. 1. SEM images of (a) $\operatorname{In}_{2} \mathrm{O}_{3} / \mathrm{rGO}$, (b) $\mathrm{SnO}_{2} / \mathrm{rGO}$, (c) $\mathrm{Fe}_{2} \mathrm{O}_{3} / \mathrm{rGO}$, and (d) $\mathrm{Co}_{3} \mathrm{O}_{4} / \mathrm{rGO}$. 


\section{The sensing properties of metal oxide/rGO hybrids}

The gas sensing properties of pristine metal oxide and metal oxide/rGO hybrids were investigated. After decorating with rGO, the sensing properties were enhanced with different fold. Especially, the optimal working temperature was reduced even to room temperature with considerable sensing sensor response. Moreover, the conductivity of rGO is much better than that of the employed metal oxide. Thus, the reasonable increase of rGO concentration could cause a decrease in the conductivity of metal oxide/rGO hybrids. Heterojunctions formed in sensing materials between different materials could account for the enhancement of gas response by promoting the adsorption of target gases (chemical sensitization) or resistance modulation upon gas adsorption (electronic sensitization). The spatially separated charge carriers above caused by heterocontacts would lead to additional modulation of the total resistance of the sensor, thus the enhanced sensing response. Furthermore, the synergetic coupling effect between metal oxide and graphene caused the extra increase of charge carrier concentration of the hybrids which leading to enhanced sensor response.
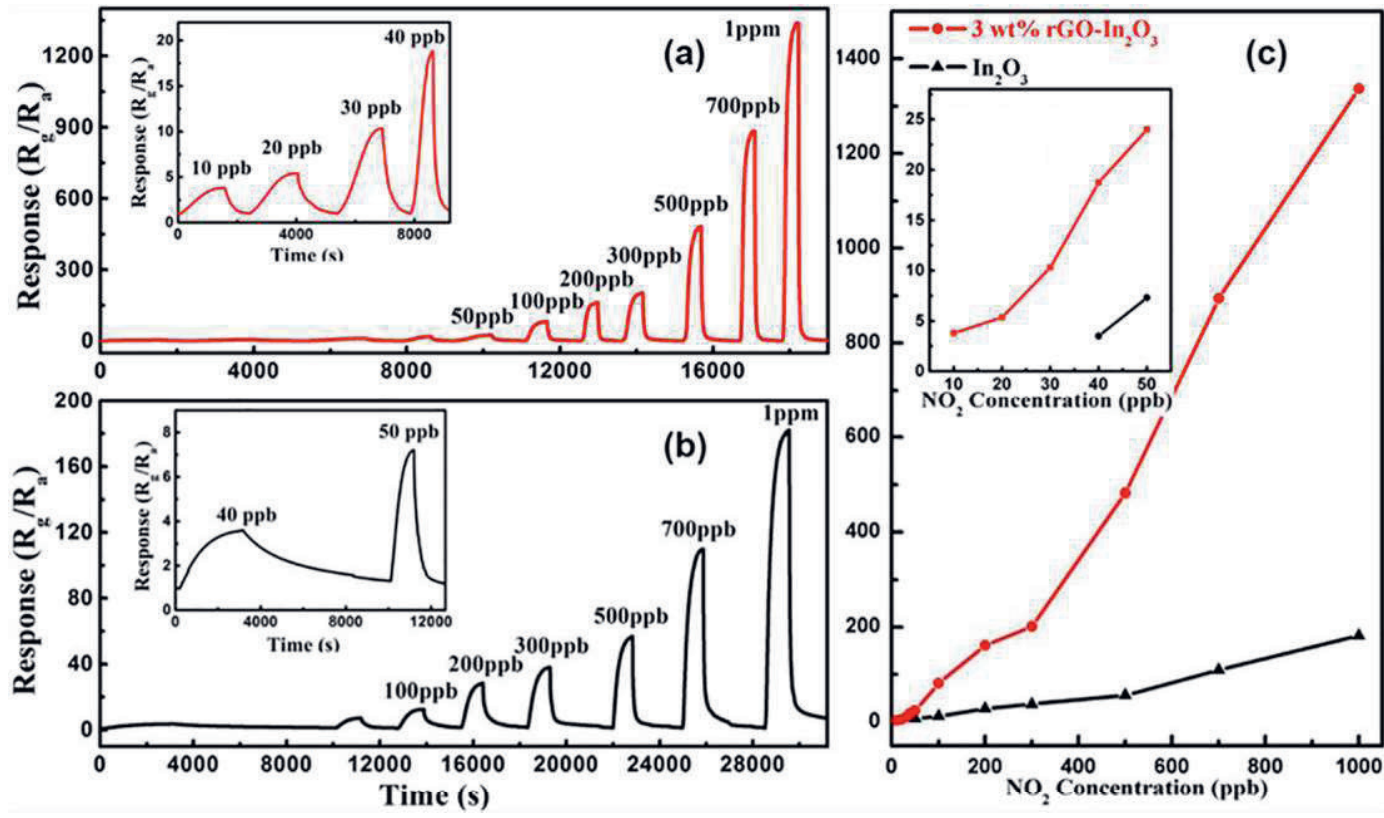

Fig. 2. Dynamic response curves to different concentrations of $\mathrm{NO}_{2}$ for the $\ln _{2} \mathrm{O}_{3}$-based and 3.0 wt $\% \ln _{2} \mathrm{O}_{3} / \mathrm{rGO}$ based sensors at respective optimal operating temperature $(a, b)$; the comparison of the response of the two sensors to the concentration of $\mathrm{NO}_{2}$ in the range of 10-1000 ppb (c).

\section{References}

[1] J. Liu, S. Li, B. Zhang, Y.L. Wang, Y. Gao, X.S. Liang, Y. Wang, G.Y. Lu, Flower-like $\ln _{2} \mathrm{O}_{3}$ modified by reduced graphene oxide sheets serving as a highly sensitive gas sensor for trace $\mathrm{NO}_{2}$ detection, Journal of Colloid and Interface Science 504, 206-213 (2017); doi: 10.1016/j.jcis.2017.05.053

[2] Y. Xiao, Q.Y. Yang, Z.Y. Wang, R. Zhang, Y. Gao, P. Sun, Y.F. Sun, G.Y. Lu, Improvement of $\mathrm{NO}_{2}$ gas sensing performance based on discoid tin oxide modified by reduced graphene oxide, Sensors and Actuators B: Chemical 227, 419-426 (2016); doi: 10.1016/j.snb.2015.11.051
[3] B. Zhang, J. Liu, X.B. Cui, Y.L. Wang, Y. Gao, P. Sun, F.M. Liu, K. Shimanoe, N. Yamazoe, G.Y. Lu, Enhanced gas sensing properties to acetone vapor achieved by $\alpha-\mathrm{Fe}_{2} \mathrm{O}_{3}$ particles ameliorated with reduced graphene oxide sheets, Sensors and Actuators B: Chemical 241, 904-914 (2017); doi: 10.1016/j.snb.2016.11.023

[4] B. Zhang, M. Cheng, G.N. Liu, Y. Gao, L.J. Zhao, S. Li, Y.P. Wang, F.M. Liu, X.S. Liang, T. Zhang, G.Y. Lu, Room temperature $\mathrm{NO}_{2}$ gas sensor based on porous $\mathrm{Co}_{3} \mathrm{O}_{4}$ slices/reduced graphene oxide hybrid, Sensors and Actuators B: Chemical 263, 387-399 (2018); doi: 10.1016/j.snb.2018.02.117 